\title{
ELECTRONIC FOREIGN-EXCHANGE MARKETS AND PASSAGE EVENTS OF INDEPENDENT SUBORDINATORS
}

\author{
MATTHIAS WINKEL, ${ }^{*}$ University of Oxford
}

\begin{abstract}
We set up a model for electronic foreign-exchange markets, suggesting subordinators to represent sellers' and buyers' offers. Its analysis naturally leads to the study of level passage events. The classical level passage event concerns the joint law of the time, height, and jump size observed when a real-valued stochastic process first exceeds a given level $h$. We provide an up-to-date treatment in the case when this process is a subordinator, and extend these results to a multivariate setting.
\end{abstract}

Keywords: Subordinator; passage time; Poisson point process technique; bid curve; ask curve; foreign-exchange market model

2000 Mathematics Subject Classification: Primary 60G51

Secondary $91 \mathrm{~B} 70$

\section{Introduction}

Modern markets, such as electronic foreign-exchange markets, collect offers to sell or buy quantities of a currency or goods at individual minimal or maximal prices. Transactions take place as soon as a buyer's price is higher than a seller's price. What remains are the unmet offers, which are commonly represented graphically as in Figure 1.

We are interested in these two price-quantity processes, which indicate to a potential buyer or seller what offers one can currently realize on the market. We propose to model these processes by subordinators, i.e. monotonic processes with stationary, independent increments. We also model the virtual parts of the processes that correspond to realized transactions, as shown in Figure 2(b) - specifically, the region $x<Q$.

Mathematically, the crossing in Figure 2(b) is related to the level passage event as studied in the late 1960s by Gusak [6] and Kesten [7] for subordinators $X \equiv\left(X_{a}\right)_{a \geq 0}$, and more general Lévy processes. These studies involved 'times'

$$
T_{h}=\inf \left\{a \geq 0: X_{a}>h\right\}, \quad h \geq 0,
$$

and their associated heights $X\left(T_{h}\right)$. Gusak's and Kesten's cumbersome approximation techniques are no longer necessary, as is seen in more recent accounts like Section III. 2 of Bertoin's book [1], where Poisson point process techniques are applied to the process of jumps of $X$. In a preliminary section, we use these techniques to provide a complete characterization of the level passage event at fixed levels $h$ or independent exponentially distributed levels $\tau$. By 'complete', we mean that we give the joint law of not only $T_{h}$ and $X\left(T_{h}\right)$, but also $X\left(T_{h}-\right)$ and,

Received 20 November 2001; revision received 3 June 2004.

* Postal address: Department of Statistics, University of Oxford, 1 South Parks Road, Oxford OX1 3TG, UK.

Email address: winkel@stats.ox.ac.uk 


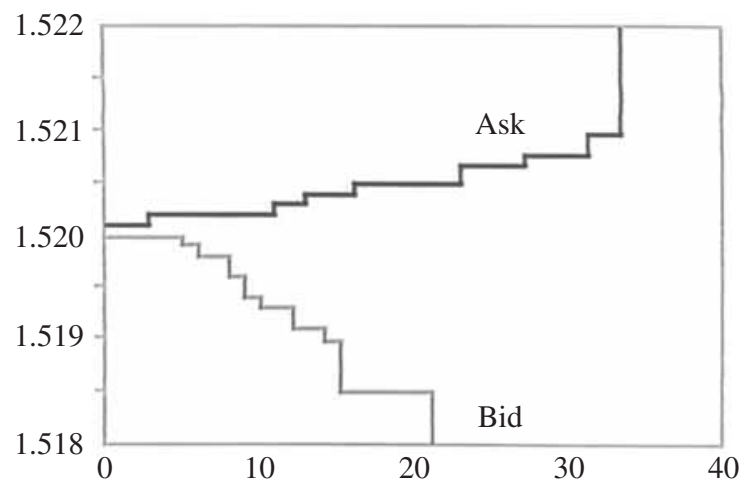

FIGURE 1: Snapshot of the DEM-USD electronic foreign-exchange market (1997), from the London School of Economics video [4] by Charles Goodhart.

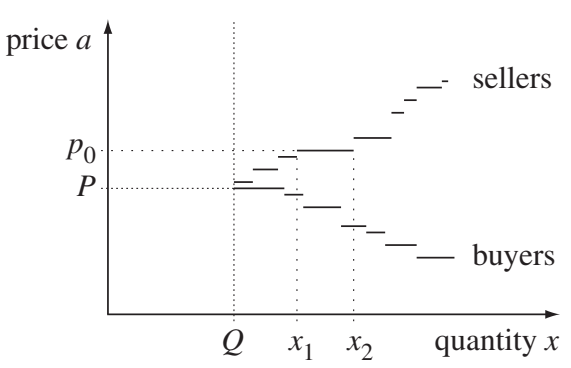

(a)

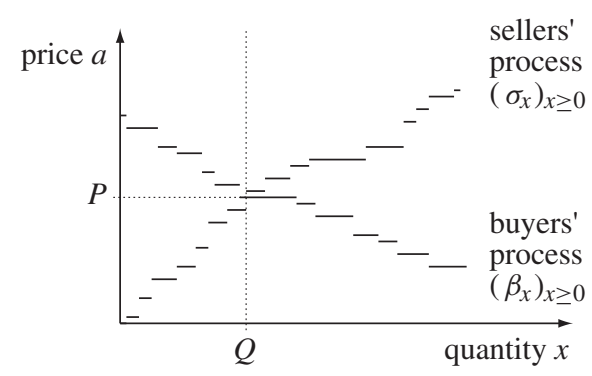

(b)

FIGURE 2: (a) Offers on the market and (b) offers plus realized transactions.

in the case of an exponential level, the level itself. Clearly, this also determines the quantities known as undershoot, overshoot, jump size at passage, etc.

The main purpose of this article is the generalization of the above to a multivariate setting. This will allow us, in Section 5, to deal with the passage of two subordinators that move towards each other as in Figure 2(b). For precise statements and derivations of the theoretical results, we refer the reader to Sections 3 and 4, in which we treat the univariate case and the passage event involving several independent subordinators, respectively. For a further extension to multivariate (dependent) subordinators with several passage events of their marginal processes, we refer to the preprint version of this article [10], which is also contained in the author's $\mathrm{PhD}$ thesis [11].

\section{Motivation: the electronic foreign-exchange market model}

Let us here describe the economic model in some more detail. Fix a given time and think of looking at a snapshot of the market. We consider the two trajectories in Figure 2(a) (and its extension to include realized transactions, as shown in Figure 2(b)) as being realizations of stochastic price processes $\left(\sigma_{x}\right)_{x \geq 0}$ and $\left(\beta_{x}\right)_{x \geq 0}$ indexed by quantity (not time, which is fixed). 
A step of $\sigma$ at height $p_{0}$ and of length $q=x_{2}-x_{1}$ corresponds to a seller who would like to sell $q$ units of the currency at a price of at least $p_{0}$, and buyers are represented similarly by the steps of $\beta$. Therefore, $\sigma_{x}$ can be seen as the price at which $x$ units are supplied to the market, while $\beta_{x}$ is the price at which $x$ units are demanded by the market.

More specifically, we can construct the processes $\sigma$ and $\beta$ as follows. Think of sellers and buyers who present their offers. Let $N_{\mathrm{S}}$ be the number of sellers; $N_{\mathrm{B}}$ the number of buyers; $P_{\mathrm{S}}(s)>0$ the minimal price at which seller $s$ would sell his $Q_{\mathrm{S}}(s)$ units of currency, $s=1, \ldots, N_{\mathrm{S}}$; and $P_{\mathrm{B}}(b)>0$ the maximal price at which a buyer $b$ would buy $Q_{\mathrm{B}}(b)$ units of currency. All information is contained in

$$
S_{a}=\sum_{\left\{s: P_{\mathrm{S}}(s) \leq a\right\}} Q_{\mathrm{S}}(s) \text { and } B_{a}=\sum_{\left\{b: P_{\mathrm{B}}(b)>a\right\}} Q_{\mathrm{B}}(b), \quad a \geq 0,
$$

the number of units offered and demanded, respectively, if the agreed price is $a$. Obviously, $S_{a}$ is increasing in $a$, starting from 0 , whereas $B_{a}$ decreases. The bid-ask equilibrium price, for which the two coincide, is

$$
P=\inf \left\{a \geq 0: S_{a}>B_{a}\right\},
$$

where it is possible that the strict inequality is replaced by a weak one or the infimum replaced by a supremum of the opposite inequality. However, we consider these differences to be negligible and will use our stated definition of the collective price $P$, which is the most suitable from a mathematical point of view.

The processes $B$ and $S$ are not precisely those whose realizations we see exemplified in diagrams similar to Figure 2(b). To make such diagrams precise, all we need to do is swap the coordinate axes, since $B$ and $S$ are quantity processes indexed by price. The corresponding price processes, indexed by quantity, are

$$
\sigma_{x}=\inf \left\{a \geq 0: S_{a}>x\right\} \quad \text { and } \quad \beta_{x}=\inf \left\{a \geq 0: B_{a}<x\right\}, \quad x \geq 0 .
$$

Let us now focus on $S$, i.e. the sellers. Without complicating the analysis, we may allow an infinite number $N_{\mathrm{S}}$ of sellers, provided that their associated quantities are summable over the appropriate price ranges in (1). As the path of a stochastic process indexed by the price parameter $a,\left(S_{a}\right)_{a \geq 0}$ is increasing and only moving in jumps, and is given here in its characterization by the point process

$$
\left\{\left(P_{\mathrm{S}}(s), Q_{\mathrm{S}}(s)\right): s=1,2, \ldots\right\}
$$

of jumps $(a, y)$ of size $y$ at price $a$. If this point process is a homogeneous (marked) Poisson point process, then $\left(S_{a}\right)_{a \geq 0}$ is a subordinator. The Poisson property implies an independence of sellers, i.e. the numbers of sellers with minimal price and quantity in disjoint regions are independent and have Poisson distributions. The homogeneity means that the law of the quantities is proportional to the Lebesgue size of a price region (in the sense of convolution powers, i.e. the quantity of currency in a region $r$ times larger than another has as its law the $r$ th convolution power of the other). It is another consequence of the homogeneous Poisson property that, conditionally on having $n$ sellers in a price-quantity region, the $n$ quantities and minimal prices are independent and identically distributed. The law of the quantities can be chosen under some consistency constraints, such as the so-called Lévy measure of the subordinator. The minimal prices have a uniform distribution on the region. Globally, this uniform spread on the price axis may seem unsatisfactory, but it is certainly acceptable locally. Note, in this context, that only minimal prices close to the actual price $P$ have a direct influence on its determination; the others only influence it by being either too low or too high. 
Next consider the buyers, as modelled by $B$. Clearly, infinitely many may occur, provided that their total quantity of currency is finite. Here, we cannot allow arbitrarily high prices for nonnegligible quantities since this makes infinite the sum defining $B$ in (1). The only possibility of allowing an infinite demand arises as the price tends to 0 , but homogeneity considerations will prohibit us from doing so. Let us look at the point process

$$
\left\{\left(P_{\mathrm{B}}(b), Q_{\mathrm{B}}(b)\right): b=1,2, \ldots\right\}
$$

of jumps $(a, y)$ of $\left(B_{a}\right)_{a \geq 0}$. Here, $(a, y)$ means a jump of size $-y$ at price $a$. If this point process is a Poisson point process, the buyers have the same independence property as the sellers. Our aim is, of course, to establish a subordinator-type property for $\left(B_{a}\right)_{a \geq 0}$, as well, but we cannot ask for homogeneity on the whole price axis $[0, \infty)$ since this would make the sums in (1) diverge almost surely. However, there are other ways to produce a subordinator setting. Mathematically, the most convenient is to fix the total quantity $B_{0}$ of currency demanded either to a constant $h>0$ or to an independent exponential random variable $\tau \equiv \tau(q)$ with parameter (inverse mean) $q>0$. Then we may consider homogeneity of the Poisson point process, but adjust the definition of $B$ to sum $Q_{\mathrm{B}}(b)$ not over all $b$ with $P_{\mathrm{B}}(b)>a$, but only over those below a random price threshold $\beta_{0}$.

We have been focusing on electronic foreign-exchange markets since they are very liquid and transparent. In this, they are an idealized version of a general market. We are modelling the fine tick-by-tick structure of these markets, which is very sensitive to the lack of transparency. The lack of liquidity may seem to be a less serious problem, but one strength of our model is its ability to deal with greatly varying offer volumes. As more and more trading is done via the internet, more and more goods fall within the range of our model.

It would be desirable to include time evolution in the (so-far) fixed-time model. Beginning with two times, the two price-quantity processes will have to be dependent for both buyers and sellers, since offers can and do remain on the market. A study of level passage events of multivariate subordinators was successfully carried out in [10], but the kind of dependence structure that emerged is not rich enough to usefully extend our market model.

There has also been empirical study and spline approximation of curves of average bid-ask prices per unit volume. We refer the reader to Gouriéroux et al. [5] and Bowsher [3].

\section{Preliminaries on the level passage event of a single one-dimensional subordinator}

Let $X \equiv\left(X_{a}\right)_{a \geq 0}$ be a subordinator, i.e. an increasing process with stationary, independent increments, whose law is represented by the Lévy-Khinchin formula for its Laplace exponent, i.e.

$$
-\frac{\ln \mathrm{E}\left(\mathrm{e}^{-q X_{a}}\right)}{a}=\Phi(q)=b q+\int_{(0, \infty)}\left(1-\mathrm{e}^{-q x}\right) \Pi(\mathrm{d} x),
$$

where we refer to $b \geq 0$ as the drift coefficient and to $\Pi$ as the Lévy measure; $\Pi$ is required to integrate $1 \wedge x$ on $(0, \infty)$. Bertoin [2] is a standard reference, as is Chapter III of Bertoin [1]. Then, $X$ has the following structure:

$$
X_{a}=b a+\sum_{0 \leq s \leq a} \Delta X_{s}, \quad a \geq 0,
$$

where $\Delta X_{a}=X_{a}-X_{a-}$, the process of jumps of $X$, is a Poisson point process with intensity measure $\Pi$. Note that the integrability condition allows $\Pi$ to be infinite 'at the origin', 
i.e. $\Pi(\varepsilon, \infty) \uparrow \infty$ as $\varepsilon \downarrow 0$. In this case, $X$ has infinitely many jumps in every interval, but these jumps are summable. If $b=0, X$ is called a pure-jump subordinator.

Independent exponential random variables $\tau \equiv \tau(\alpha) \sim \operatorname{Exp}(\alpha)$ are often very useful. An example of their occurrence is in resolvents

$$
\begin{aligned}
V^{\alpha}(\mathrm{d} x) & =\int_{0}^{\infty} \mathrm{e}^{-\alpha a} \mathrm{P}\left(X_{a} \in \mathrm{d} x\right) \mathrm{d} a \quad(\alpha \geq 0) \\
& =\frac{1}{\alpha} \mathrm{P}\left(X_{\tau(\alpha)} \in \mathrm{d} x\right) \quad(\text { if } \alpha>0) .
\end{aligned}
$$

It is an immediate consequence of this that their Laplace transforms are $(\alpha+\Phi(q))^{-1}$. We will meet resolvents in the context of hitting times

$$
H_{h}=\inf \left\{a \geq 0: X_{a}=h\right\} \in(0, \infty], \quad h \geq 0 .
$$

If $b=0$ then $H_{h}=\infty$ almost surely for almost all $h \geq 0$ (there are countably many exceptions if $\Pi$ is finite and has atoms, i.e. points $x$ at which $\Pi(\{x\})>0)$. If $b>0$ then $V^{\alpha}$ admits a density $v^{\alpha}$, with respect to the Lebesgue measure, which is continuous on $(0, \infty)$ and satisfies $v^{\alpha}(0)=v^{\alpha}(0+)=1 / b$. Furthermore,

$$
\mathrm{E}\left(\exp \left\{-\alpha H_{h}\right\}\right)=b v^{\alpha}(h)
$$

cf. Corollary II.18 and Theorem III.5 of [1].

Concerning the passage of a level $h \geq 0$, three basic and three associated quantities are of interest; namely the passage time $T_{h}$, the passage height $X\left(T_{h}\right)$ and the jump size $\Delta X\left(T_{h}\right)$ of the level passage, the overshoot $o_{h}$, the undershoot $u_{h}$ and the prepassage height $X\left(T_{h}-\right)$. Mathematically, these are

$$
\begin{aligned}
T_{h} & =\inf \left\{a \geq 0: X_{a}>h\right\}, \\
o_{h} & =X\left(T_{h}\right)-h, \\
u_{h} & =h-X\left(T_{h}-\right), \\
\Delta X\left(T_{h}\right) & =X\left(T_{h}\right)-X\left(T_{h}-\right)=o_{h}+u_{h} \equiv \Delta_{h} .
\end{aligned}
$$

If $X$ is almost surely strictly increasing, i.e. if $\Pi$ is an infinite measure or $b>0$, then almost surely for all $h \geq 0, T_{h}$ coincides with $\inf \left\{a \geq 0: X_{a} \geq h\right\}$.

We will also treat the situation in which $h$ is replaced by an independent exponential height $\tau \equiv \tau(q)$ with parameter $q>0$. Then we can give the joint law of these quantities in terms of the Laplace exponent $\Phi$ of $X$. The central result of this section is the following. It is stated in three different ways: (a) as a distributional identity for a fixed level $h$; (b) as a distributional identity for an exponential level $\tau(q)$; and (c) as a Laplace transform identity for an exponential level, which can also be viewed as a double Laplace transform of the fixed-level distributions.

Theorem 1. (a) For all $h>0$,

$$
\begin{aligned}
& \mathrm{P}\left(T_{h} \in \mathrm{d} a, X\left(T_{h}-\right) \in \mathrm{d} y, \Delta_{h} \in \mathrm{d} z\right) \\
& \quad=\delta_{h}(\mathrm{~d} y) \mathrm{P}\left(H_{h} \in \mathrm{d} a\right) \delta_{0}(\mathrm{~d} z)+\mathbf{1}_{\{0 \leq y \leq h<y+z\}} \mathrm{P}\left(X_{a} \in \mathrm{d} y\right) \mathrm{d} a \Pi(\mathrm{d} z),
\end{aligned}
$$

where $\delta_{h}$ denotes a Dirac point mass in $h$. 
(b) For all $q>0$,

$$
\begin{aligned}
& \mathrm{P}\left(T_{\tau(q)} \in \mathrm{d} a, X\left(T_{\tau(q)}-\right) \in \mathrm{d} y, \Delta_{\tau(q)} \in \mathrm{d} z\right) \\
& \quad=\mathrm{P}\left(H_{y} \in \mathrm{d} a\right) q \mathrm{e}^{-q y} \mathrm{~d} y \delta_{0}(\mathrm{~d} z)+\mathrm{e}^{-q y} \mathrm{P}\left(X_{a} \in \mathrm{d} y\right) \mathrm{d} a\left(1-\mathrm{e}^{-q z}\right) \Pi(\mathrm{d} z) .
\end{aligned}
$$

(c) For all $q>0, \kappa>-q, \lambda>-q-\kappa, \alpha>-\Phi(q+\kappa+\lambda)$, and $v \geq 0$,

$$
\mathrm{E}\left(\exp \left\{-\kappa \tau(q)-\alpha T_{\tau(q)}-\lambda X\left(T_{\tau(q)}-\right)-v \Delta_{\tau(q)}\right\}\right)=\frac{q(\Phi(q+\kappa+v)-\Phi(v))}{(q+\kappa)(\alpha+\Phi(q+\kappa+\lambda))} .
$$

Proof. (a) We first generalize the argument in Proposition III.2 of [1] to include the time component. That argument, using the Poisson point process technique, yields

$$
\mathrm{P}\left(T_{h} \in \mathrm{d} a, X\left(T_{h}-\right) \in \mathrm{d} y, \Delta_{h} \in \mathrm{d} z\right)=\mathrm{P}\left(X_{a} \in \mathrm{d} y\right) \mathrm{d} a \Pi(\mathrm{d} z)
$$

when $0 \leq y \leq h<y+z$ and $0<a<\infty$. The full argument can also be found as a special case in the proofs of generalizations that appear below.

The term for $z=0$ is obtained as follows:

$$
\begin{aligned}
\mathrm{P}\left(T_{h} \in \mathrm{d} a, X\left(T_{h}-\right) \in \mathrm{d} y, \Delta_{h}=0\right) & =\delta_{h}(y) \mathrm{P}\left(T_{h} \in \mathrm{d} a, X\left(T_{h}\right)=h\right) \\
& =\delta_{h}(y) \mathrm{P}\left(H_{h} \in \mathrm{d} a\right) .
\end{aligned}
$$

(b) We calculate, for bounded real functions $f, g$, and $\ell$, that

$$
\begin{aligned}
& \mathrm{E}\left(f\left(T_{\tau}\right) g\left(X\left(T_{\tau}-\right)\right) \ell\left(\Delta_{\tau}\right)\right) \\
&=\int_{0}^{\infty} q \mathrm{e}^{-q h} \int_{[0, \infty)} f(a) g(h) \ell(0) \mathrm{P}\left(H_{h} \in \mathrm{d} a\right) \mathrm{d} h \\
& \quad+\int_{0}^{\infty} q \mathrm{e}^{-q h} \int_{0}^{\infty} \int_{[0, h]} \int_{(h-y, \infty)} f(a) g(y) \ell(z) \Pi(\mathrm{d} z) \mathrm{P}\left(X_{a} \in \mathrm{d} y\right) \mathrm{d} a \mathrm{~d} h \\
&= \int_{0}^{\infty} \int_{[0, \infty)} f(a) g(y) \ell(0) \mathrm{P}\left(H_{y} \in \mathrm{d} a\right) q \mathrm{e}^{-q y} \mathrm{~d} y \\
&+\int_{(0, \infty)} \int_{0}^{\infty} \int_{[0, \infty)} f(a) g(y) \ell(z) \mathrm{e}^{-q y}\left(1-\mathrm{e}^{-q z}\right) \mathrm{P}\left(X_{a} \in \mathrm{d} y\right) \mathrm{d} a \Pi(\mathrm{d} z) .
\end{aligned}
$$

(c) We deduce this result from part (a), by integration. First, assume that $\kappa=0$. Then, from (3) and (4), we obtain

$$
\begin{aligned}
\mathrm{E}\left(\exp \left\{-\alpha T_{\tau}-\lambda X\left(T_{\tau}\right)-v \Delta_{\tau}\right\} \mathbf{1}_{\left\{\Delta_{\tau}=0\right\}}\right) & =\int_{0}^{\infty} q \mathrm{e}^{-(q+\lambda) h} \mathrm{E}\left(\mathrm{e}^{-\alpha H_{h}}\right) \mathrm{d} h \\
& =\frac{b q}{\alpha+\Phi(q+\lambda)} .
\end{aligned}
$$


The calculation of the second term involves Fubini's theorem and the Lévy-Khinchin representation (2) only:

$$
\begin{aligned}
& \mathrm{E}\left(\exp \left\{-\alpha T_{\tau}-\lambda X\left(T_{\tau}\right)-v \Delta_{\tau}\right\} \mathbf{1}_{\left\{\Delta_{\tau}>0\right\}}\right) \\
& =\int_{0}^{\infty} q \mathrm{e}^{-q h} \int_{0}^{\infty} \mathrm{e}^{-\alpha a} \int_{[0, h]} \mathrm{e}^{-\lambda y} \int_{(h-y, \infty)} \mathrm{e}^{-v z} \Pi(\mathrm{d} z) \mathrm{P}\left(X_{a} \in \mathrm{d} y\right) \mathrm{d} a \mathrm{~d} h \\
& =\int_{0}^{\infty} \mathrm{e}^{-\alpha a} \int_{[0, \infty)} \mathrm{e}^{-\lambda y} \int_{(0, \infty)} \mathrm{e}^{-\nu z} \int_{y}^{y+z} q \mathrm{e}^{-q h} \mathrm{~d} h \Pi(\mathrm{d} z) \mathrm{P}\left(X_{a} \in \mathrm{d} y\right) \mathrm{d} a \\
& =\frac{\Phi(q+v)-\Phi(\nu)-b q}{\alpha+\Phi(q+\lambda)} .
\end{aligned}
$$

Finally, we deduce that

$$
\begin{aligned}
& \mathrm{E}\left(\exp \left\{-\kappa \tau(q)-\alpha T_{\tau(q)}-\lambda X\left(T_{\tau(q)}-\right)-v \Delta_{\tau(q)}\right\}\right) \\
& \quad=q \int_{0}^{\infty} \mathrm{e}^{-q h} \mathrm{e}^{-\kappa h} \mathrm{E}\left(\exp \left\{-\alpha T_{h}-\lambda X\left(T_{h}-\right)-v \Delta_{h}\right\}\right) \mathrm{d} h \\
& =\frac{q}{q+\kappa} \mathrm{E}\left(\exp \left\{-\alpha T_{\tau(q+\kappa)}-\lambda X\left(T_{\tau(q+\kappa)}-\right)-v \Delta_{\tau(q+\kappa)}\right\}\right) \\
& =\frac{q(\Phi(q+\kappa+v)-\Phi(v))}{(q+\kappa)(\alpha+\Phi(q+\kappa+\lambda))} .
\end{aligned}
$$

This completes the proof.

The complete joint law of all involved quantities now follows as a corollary. From this, we can easily obtain concise formulae for the laws of any selection of marginals.

Corollary 1. (i) We have, for all $q, \kappa, \alpha, \beta, \gamma, \lambda, \mu$, and $v$ such that the right-hand side of the following formula is defined,

$$
\begin{aligned}
\mathrm{E}\left(\operatorname { e x p } \left\{-\kappa \tau-\alpha T_{\tau}-\beta u_{\tau}-\gamma o_{\tau}-\lambda X\right.\right. & \left.\left.\left(T_{\tau}-\right)-\mu X\left(T_{\tau}\right)-v \Delta_{\tau}\right\}\right) \\
& =\frac{q(\Phi(q+\kappa+\mu+\beta+\nu)-\Phi(\gamma+\mu+v))}{(q+\kappa+\beta-\gamma)(\alpha+\Phi(q+\kappa+\mu+\lambda))} .
\end{aligned}
$$

(ii) In particular, $T_{\tau} \sim \operatorname{Exp}(\Phi(q))$ and $\left(T_{\tau}, X\left(T_{\tau}-\right)\right)$ is independent of $\left(u_{\tau}, o_{\tau}, \Delta_{\tau}\right)$.

The joint distribution of $\left(T_{h}, X\left(T_{h}\right)\right)$ is originally due to Gusak [6] and Kesten [7], even in a more general Lévy process setting. For related results at exponential heights, cf. Exercise VI.1, Theorem VII.4 of Bertoin [1] and Lemma 1.11 of [2].

The independence of $\left(T_{\tau}, X\left(T_{\tau}-\right)\right)$ and $\left(u_{\tau}, o_{\tau}\right)$ can be strengthened as follows.

Proposition 1. $\left(X_{a}\right)_{0 \leq a<T_{\tau}}$ is independent of $\left(u_{\tau}, o_{\tau}\right)$.

Proof. This is once more due to the Poisson point process property of the process $J_{a}=$ $X_{a}-X_{a-}$ of jumps of $X$. Let $\beta>0$ and $\gamma>0$, and choose an arbitrary functional $f$ on the 
path space. If $b=0$ then

$$
\begin{aligned}
& \mathrm{E}(\left.f\left(\left(X_{s}\right)_{0 \leq s<T_{\tau}}\right) \exp \left\{-\beta u_{\tau}-\gamma o_{\tau}\right\}\right) \\
&=\mathrm{E}\left(\sum_{a \geq 0} f\left(\left(X_{S}\right)_{0 \leq s<a}\right) \exp \left\{-\beta\left(\tau-X_{a-}\right)-\gamma\left(X_{a-}+J_{a}-\tau\right)\right\} \mathbf{1}_{\left\{X_{a-} \leq x, J_{a}>\tau-X_{a-}\right\}}\right) \\
&=\int_{0}^{\infty} \mathrm{E}\left(f\left(\left(X_{s}\right)_{0 \leq s<a}\right) \mathbf{1}_{\left\{X_{a-} \leq \tau\right\}}\right. \\
&\left.\quad \times \int_{(0, \infty)} \exp \left\{-\beta\left(\tau-X_{a-}\right)-\gamma\left(X_{a-}+z-\tau\right)\right\} \mathbf{1}_{\left\{z>\tau-X_{a-}\right\}} \Pi(\mathrm{d} z)\right) \mathrm{d} a \\
&=\int_{0}^{\infty} \int_{[0, \infty)} \mathrm{E}\left(f\left(\left(X_{s}\right)_{0 \leq s<a}\right) \mid X_{a-}=y\right) \mathrm{e}^{-(\gamma-\beta) y} \\
& \quad \times \int_{(0, \infty)} \mathrm{e}^{-\gamma z} \int_{y}^{y+z} q \mathrm{e}^{-q h} \mathrm{e}^{-(\beta-\gamma) h} \mathrm{~d} h \Pi(\mathrm{d} z) \mathrm{P}\left(X_{a-} \in \mathrm{d} y\right) \mathrm{d} a \\
&=\left(\int_{0}^{\infty} \int_{[0, \infty)} \mathrm{E}\left(f\left(\left(X_{s}\right)_{0 \leq s<a}\right) \mid X_{a-}=y\right) \mathrm{e}^{-q y} \mathrm{P}\left(X_{a-} \in \mathrm{d} y\right) \mathrm{d} a\right) \\
& \quad \times \frac{q}{q+\beta-\gamma} \int_{(0, \infty)}\left(\left(1-\mathrm{e}^{-(q+\beta) z}\right)-\left(1-\mathrm{e}^{-\gamma z}\right)\right) \Pi(\mathrm{d} z),
\end{aligned}
$$

which shows the asserted independence. If $b>0$, denoting the range of $X$ by $\mathcal{R}$, we obtain from the second line an additional term - cf. Corollary IV.6 of [1] -

$$
\begin{aligned}
\mathrm{E}\left(f\left(\left(X_{s}\right)_{0 \leq s<T_{\tau}}\right) \mathbf{1}_{\left\{\Delta_{\tau}=0\right\}}\right) & =\int_{0}^{\infty} q \mathrm{e}^{-q h} \mathrm{E}\left(f\left(\left(X_{s}\right)_{0 \leq s<T_{h}}\right) \mathbf{1}_{\{h \in \mathcal{R}\}}\right) \mathrm{d} h \\
& =\mathrm{E}\left(\int_{0}^{\infty} f\left(\left(X_{s}\right)_{0 \leq s<T_{h}}\right) \mathrm{e}^{-q X\left(T_{h}-\right)} q b \mathrm{~d} T_{h}\right) \\
& =\mathrm{E}\left(\int_{0}^{\infty} f\left(\left(X_{s}\right)_{0 \leq s<a}\right) \mathrm{e}^{-q X_{a-}} q b \mathrm{~d} a\right) \\
& =q b \int_{0}^{\infty} \mathrm{E}\left(f\left(\left(X_{s}\right)_{0 \leq s<a}\right) \mathrm{e}^{-q X_{a-}}\right) \mathrm{d} a
\end{aligned}
$$

which cancels with an additional term occurring in the last line of the preceding equation, due to the fact that

$$
\int_{(0, \infty)}\left(\left(1-\mathrm{e}^{-(q+\beta) z}\right)-\left(1-\mathrm{e}^{-\gamma z}\right)\right) \Pi(\mathrm{d} z)=\Phi(q+\beta)-\Phi(\gamma)-b(q+\beta-\gamma) .
$$

This completes the proof.

\section{Level passage for a sum of independent subordinators}

Let $X=X^{(0)}+X^{(1)}+\cdots+X^{(m)}$ be a subordinator built from a deterministic drift $X_{a}^{(0)}=$ $b a$, and $m$ independent pure-jump subordinators $X^{(1)}, \ldots, X^{(m)}$. We study

$$
T_{h}=\inf \left\{a \geq 0: X_{a}>h\right\}, \quad h \geq 0,
$$

the associated individual heights $X^{(i)}\left(T_{h}-\right), i=1, \ldots, m$, and the jump occurring at $T_{h}$ - there is at most only one jump since independent subordinators have no common jump times almost 
surely; cf. Proposition XII.(1.5) of [8], for example. We use the obvious indexed notation for the Laplace exponent, Lévy measure, etc., of $X^{(i)}, i=0, \ldots, m$. Clearly,

$$
\Phi(q)=\sum_{i=0}^{m} \Phi^{(i)}(q) \quad \text { and } \quad \Pi(\mathrm{d} z)=\sum_{i=1}^{m} \Pi^{(i)}(\mathrm{d} z) .
$$

\subsection{Which subordinator performs the passage?}

In this subsection, we will not concern ourselves with the laws of the quantities introduced but only with how the passage takes place. Specifically, we calculate the probabilities that the drift $(i=0)$ or a jump $(i=1, \ldots, m)$ of $X^{(i)}$ makes $X$ pass the level. We denote these events by

$$
A^{(0)}(h)=\left(\bigcup_{i=1}^{m} A^{(i)}(h)\right)^{\mathrm{c}}, \quad A^{(i)}(h)=\left\{\Delta X^{(i)}\left(T_{h}\right)>0\right\}, \quad i=1, \ldots, m .
$$

The 0-resolvent measure $V=V^{0}$ of $X-$ cf. (3) - is called the renewal measure. Recall that $b>0$ entails the existence of a Lebesgue density $v$ that is continuous on $(0, \infty)$. We also introduce the tails $\bar{\Pi}^{(i)}(x)=\Pi^{(i)}(x, \infty)$ of the Lévy measures of $X^{(i)}$.

Proposition 2. (a) For all $h>0$,

$$
\mathrm{P}\left(A^{(0)}(h)\right)=b v(h) \quad \text { and } \quad \mathrm{P}\left(A^{(i)}(h)\right)=\bar{\Pi}^{(i)} * V(h), \quad i=1, \ldots, m,
$$

where $*$ is the convolution of a function with a measure.

(b) For $\tau=\tau(q), q>0$,

$$
\mathrm{P}\left(A^{(i)}(\tau)\right)=\frac{\Phi^{(i)}(q)}{\Phi(q)}, \quad i=0, \ldots, m .
$$

Proof. (a) The first expression is the probability that $X$ hits $h$; cf. (4). For the second probability, we repeat the proof of Proposition III.2 of [1] to obtain, for $0 \leq x \leq h<x+z$,

$$
\mathrm{P}\left(X\left(T_{h}-\right) \in \mathrm{d} x, X^{(i)}\left(T_{h}\right)-X^{(i)}\left(T_{h}-\right) \in \mathrm{d} z\right)=\Pi^{(i)}(\mathrm{d} z) V(\mathrm{~d} x)
$$

and, therefore,

$$
\mathrm{P}\left(X^{(i)}\left(T_{h}\right)-X^{(i)}\left(T_{h}-\right)>0\right)=\int_{[0, h]} \bar{\Pi}^{(i)}(h-x) V(\mathrm{~d} x)=\bar{\Pi}^{(i)} * V(h) .
$$

(b) We integrate the formulae in part (a) with respect to the exponential law and use the facts that the Laplace transforms of $V$ and $\bar{\Pi}^{(i)}(h) \mathrm{d} h$ are $1 / \Phi(q)$ and $\Phi^{(i)}(q) / q$, respectively; see Section III.1 of [1].

The special case $m=2$ can be interpreted differently. Also, we see that our supposition that the only drift term is in $X^{(0)}$ is for notational ease.

Example 1. (Passage of two subordinators moving towards each other.) Let $Y$ and $Z$ be two independent subordinators with drift coefficient $b_{Y+Z}$, Lévy measures $\Pi_{Y}$ and $\Pi_{Z}$, etc., associated using the obvious notation. We consider $Y$, as described, and $h-Z$, which is the subordinator starting from $h$ and moving downwards, towards $Y$. The passage event of 
$X=Y+Z$ over level $h$ is the event that $Y$ and $h-Z$ cross. The preceding proposition yields

(a) for all $h>0$,

$$
\begin{aligned}
& \mathrm{P}\left(A_{0}(h)\right)=\mathrm{P}\left(Y\left(T_{h}-\right)=Y\left(T_{h}\right)=h-Z\left(T_{h}\right)=h-Z\left(T_{h}-\right)\right)=b_{Y+Z} v_{Y+Z}(h), \\
& \mathrm{P}\left(A_{Y}(h)\right)=\mathrm{P}\left(Y\left(T_{h}-\right)<h-Z\left(T_{h}\right)=h-Z\left(T_{h}-\right)<Y\left(T_{h}\right)\right)=\bar{\Pi}_{Y} * V_{Y+Z}(h), \\
& \mathrm{P}\left(A_{Z}(h)\right)=\mathrm{P}\left(h-Z\left(T_{h}\right)<Y\left(T_{h}-\right)=Y\left(T_{h}\right)<h-Z\left(T_{h}-\right)\right)=\bar{\Pi}_{Z} * V_{Y+Z}(h) ;
\end{aligned}
$$

and

(b) for $\tau=\tau(q), q>0$,

$$
\begin{aligned}
& \mathrm{P}\left(A_{0}(\tau)\right)=\mathrm{P}\left(Y\left(T_{\tau}-\right)=Y\left(T_{\tau}\right)=\tau-Z\left(T_{\tau}\right)=\tau-Z\left(T_{\tau}-\right)\right)=\frac{q b_{Y+Z}}{\Phi_{Y+Z}(q)}, \\
& \mathrm{P}\left(A_{Y}(\tau)\right)=\mathrm{P}\left(Y\left(T_{\tau}-\right)<\tau-Z\left(T_{\tau}\right)=\tau-Z\left(T_{\tau}-\right)<Y\left(T_{\tau}\right)\right)=\frac{\Phi_{Y-b_{Y} I}(q)}{\Phi_{Y+Z}(q)}, \\
& \mathrm{P}\left(A_{Y}(\tau)\right)=\mathrm{P}\left(\tau-Z\left(T_{\tau}\right)<Y\left(T_{\tau}-\right)=Y\left(T_{\tau}\right)<\tau-Z\left(T_{\tau}-\right)\right)=\frac{\Phi_{Z-b_{Z} I}(q)}{\Phi_{Y+Z}(q)},
\end{aligned}
$$

where $I$ denotes the identity process $I_{t}=t$.

\subsection{The joint law of the passage variables}

We now turn to the joint law of the involved quantities. These are principally the passage time $T_{h}$, the prepassage heights $X^{(i)}\left(T_{h}-\right), i=1, \ldots, m$, the jump size $\Delta_{h}$, and the information about which subordinator performs the passage, i.e. which of the events $A^{(i)}$, $i=0, \ldots, m$, occurs. When $h$ is replaced by an independent exponential height $\tau$, this quantity is also added to the list. Note, however, that on $A^{(0)}$ there is no jump at time $T_{\tau}$ and, hence, $\tau=X^{(0)}\left(T_{\tau}-\right)+\cdots+X^{(m)}\left(T_{\tau}-\right)=X^{(0)}\left(T_{\tau}\right)+\cdots+X^{(m)}\left(T_{\tau}\right)$ is determined by $X^{(0)}\left(T_{\tau}-\right)=b T_{\tau}, X^{(1)}\left(T_{\tau}-\right), \ldots, X^{(m)}\left(T_{\tau}-\right)$. From these principal quantities, we can derive, e.g. the overshoot, undershoot, and passage heights by linear transformations.

In this setting, we formulate the main theorem of this section.

Theorem 2. (a) For all $h>0$ and $j=1, \ldots, m$,

$$
\begin{aligned}
\mathrm{P}\left(T_{h}\right. & \left.\in \mathrm{d} a, X^{(i)}\left(T_{h}-\right) \in \mathrm{d} x_{i}, i=1, \ldots, m, \Delta_{h} \in \mathrm{d} z, A^{(j)}\right) \\
& =\mathbf{1}_{\left\{x_{1}+\cdots+x_{m} \leq h<x_{1}+\cdots+x_{m}+z\right\}}\left(\prod_{i=1}^{m} \mathrm{P}\left(X_{a}^{(i)} \in \mathrm{d} x_{i}\right)\right) \mathrm{d} a \Pi^{(j)}(\mathrm{d} z) .
\end{aligned}
$$

Furthermore, if $b>0$ then

$$
\begin{aligned}
& \mathrm{P}\left(T_{h} \in \mathrm{d} a, X^{(i)}\left(T_{h}-\right) \in \mathrm{d} x_{i}, i=1, \ldots, m, \Delta_{h} \in \mathrm{d} z, A^{(0)}\right) \\
& \quad=\mathrm{P}\left(X_{a}^{(i)} \in \mathrm{d} x_{i}, i=1, \ldots, m \mid X_{a}=h\right) \mathrm{P}\left(H_{h} \in \mathrm{d} a\right) \delta_{0}(\mathrm{~d} z) .
\end{aligned}
$$

(b) For all $q>0$ and $j=1, \ldots, m$,

$$
\begin{aligned}
\mathrm{P}\left(T_{\tau}\right. & \left.\in \mathrm{d} a, X^{(i)}\left(T_{\tau}-\right) \in \mathrm{d} x_{i}, i=1, \ldots, m, \Delta_{\tau} \in \mathrm{d} z, A^{(0)}\right) \\
& =\left(\prod_{i=1}^{m} \mathrm{e}^{-q x_{i}} \mathrm{P}\left(X_{a}^{(i)} \in \mathrm{d} x_{i}\right)\right) \mathrm{d} a b q \delta_{0}(\mathrm{~d} z)
\end{aligned}
$$


and

$$
\begin{aligned}
\mathrm{P}\left(T_{\tau}\right. & \left.\in \mathrm{d} a, X^{(i)}\left(T_{\tau}-\right) \in \mathrm{d} x_{i}, i=1, \ldots, m, \Delta_{\tau} \in \mathrm{d} z, A^{(j)}\right) \\
& =\left(\prod_{i=1}^{m} \mathrm{e}^{-q x_{i}} \mathrm{P}\left(X_{a}^{(i)} \in \mathrm{d} x_{i}\right)\right) \mathrm{d} a\left(1-\mathrm{e}^{-q z}\right) \Pi^{(j)}(\mathrm{d} z) .
\end{aligned}
$$

(c) For all $q>0, \kappa \geq 0, \alpha \geq 0, \xi_{1} \geq 0, \ldots, \xi_{m} \geq 0, v \geq 0$, and $j=0, \ldots, m$,

$$
\begin{gathered}
\mathrm{E}\left(\exp \left\{-\kappa \tau-\alpha T_{\tau}-\xi_{1} X^{(1)}\left(T_{\tau}-\right)-\cdots-\xi_{m} X^{(m)}\left(T_{\tau}-\right)-v \Delta_{\tau}\right\} \mathbf{1}_{A^{(j)}}\right) \\
=\frac{q\left(\Phi^{(j)}(q+\kappa+v)-\Phi^{(j)}(v)\right)}{(q+\kappa)\left(\alpha+\Phi^{(1)}\left(q+\kappa+\xi_{1}\right)+\cdots+\Phi^{(m)}\left(q+\kappa+\xi_{m}\right)\right)} .
\end{gathered}
$$

Proof. (a) The second law is immediate since either $b=0$ or $\left\{H_{h}=a\right\}=\left\{X_{a}=h\right\}$ for all $h>0$ and $a>0$. The first is obtained, as in Theorem 1(b), by verifying, via the compensation formula for the Poisson point process $J_{a}^{(j)}=X_{a}^{(j)}-X_{a-}^{(j)}$ of jumps of $X^{(j)}$ (which ignores the jumps of $X^{(i)}$ for $i \neq j$ ) that, for $f, g$, and $\ell$ bounded measurable functions,

$$
\begin{aligned}
\mathrm{E}\left(f\left(T_{h}\right) g\left(X^{(1)}\left(T_{h}-\right), \ldots, X^{(m)}\left(T_{h}-\right)\right) \ell\left(\Delta_{h}\right) \mathbf{1}_{A^{(j)}}\right) \\
\quad=\mathrm{E}\left(\sum_{a \geq 0} f(a) g\left(X_{a-}^{(1)}, \ldots, X_{a-}^{(m)}\right) \ell\left(J_{a}^{(j)}\right) \mathbf{1}_{\left\{X_{a-} \leq h, J_{a}>h-X_{a-\}}\right\}}\right) \\
\quad=\int_{0}^{\infty} f(a) \mathrm{E}\left(g\left(X_{a-}^{(1)}, \ldots, X_{a-}^{(m)}\right) \mathbf{1}_{\left\{X_{a-} \leq h\right\}} \int_{(0, \infty)} \ell(z) \mathbf{1}_{\left\{z>h-X_{a-\}}\right.} \Pi^{(j)}(\mathrm{d} z)\right) \mathrm{d} a \\
=\int_{0}^{\infty} \int_{\substack{0 \leq x_{1}+\cdots+x_{m} \\
\leq h<x_{1}+\cdots+x_{m}+z}} f(a) g\left(x_{1}, \ldots, x_{m}\right) \ell(z)\left(\prod_{i=1}^{m} \mathrm{P}\left(X_{a}^{(i)} \in \mathrm{d} x_{i}\right)\right) \Pi^{(j)}(\mathrm{d} z) \mathrm{d} a .
\end{aligned}
$$

(b) We verify the first law by fixing arbitrary bounded measurable functions $f$ and $g$ and using Corollary IV.6 of [1], as in the proof of Proposition 1, above:

$$
\begin{aligned}
\mathrm{E}(f( & \left.\left.T_{\tau}\right) g\left(X^{(1)}\left(T_{\tau}-\right), \ldots, X^{(m)}\left(T_{\tau}-\right)\right) \mathbf{1}_{\left\{X\left(T_{\tau}-\right)=\tau\right\}}\right) \\
& =\int_{0}^{\infty} q \mathrm{e}^{-q h} \mathrm{E}\left(f\left(T_{h}\right) g\left(X^{(1)}, \ldots, X^{(m)}\left(T_{h}-\right)\right) \mathbf{1}_{\left\{X\left(T_{h}-\right)=h\right\}}\right) \mathrm{d} h \\
& =\mathrm{E}\left(\int_{0}^{\infty} q \mathrm{e}^{-q X\left(T_{h}-\right)} f\left(T_{h}\right) g\left(X^{(1)}\left(T_{h}-\right), \ldots, X^{(m)}\left(T_{h}-\right)\right) \mathbf{1}_{\{h \in \mathcal{R}\}} \mathrm{d} h\right) \\
& =\mathrm{E}\left(\int_{0}^{\infty} q \mathrm{e}^{-q X\left(T_{h}-\right)} f\left(T_{h}\right) g\left(X^{(1)}\left(T_{h}-\right), \ldots, X^{(m)}\left(T_{h}-\right)\right) b \mathrm{~d} T_{h}\right) \\
& =\mathrm{E}\left(\int_{0}^{\infty} q \mathrm{e}^{-q X_{a-}} f(a) g\left(X_{a-}^{(1)}, \ldots, X_{a-}^{(m)}\right) b \mathrm{~d} a\right) \\
& =\int_{0}^{\infty} \mathrm{E}\left(q \mathrm{e}^{-q X_{a-}} f(a) g\left(X_{a-}^{(1)}, \ldots, X_{a-}^{(m)}\right)\right) b \mathrm{~d} a \\
& =\int_{0}^{\infty} \int_{[0, \infty)^{m}} f(a) g\left(x_{1}, \ldots, x_{m}\right) q b \mathrm{e}^{-q\left(x_{1}+\cdots+x_{m}\right)}\left(\prod_{i=1}^{m} \mathrm{P}\left(X_{a}^{(i)} \in \mathrm{d} x_{i}\right)\right) \mathrm{d} a .
\end{aligned}
$$

The second formula follows from part (a), by Fubini's theorem. 
(c) From part (b), we calculate, for $j=0$, that

$$
\begin{aligned}
\mathrm{E}\left(\exp \left\{-\alpha T_{\tau}-\xi_{1} X^{(1)}\left(T_{\tau}-\right)-\cdots-\xi_{m} X^{(m)}\left(T_{\tau}-\right)-\nu \Delta_{\tau}\right\} \mathbf{1}_{A^{(0)}}\right) \\
=\int_{0}^{\infty} \int_{[0, \infty)^{m}} q \mathrm{e}^{-q\left(x_{1}+\cdots+x_{m}\right)} \mathrm{e}^{-\alpha a-\xi_{1} x_{1}-\cdots-\xi_{m} x_{m}} \\
\quad \times \mathrm{P}\left(X_{a}^{(1)} \in \mathrm{d} x_{1}\right) \cdots \mathrm{P}\left(X_{a}^{(m)} \in \mathrm{d} x_{m}\right) b \mathrm{~d} a \\
=\int_{0}^{\infty} \exp \left\{-\left(\alpha+\Phi^{(1)}\left(q+\xi_{1}\right)+\cdots+\Phi^{(m)}\left(q+\xi_{m}\right)\right) a\right\} q b \mathrm{~d} a \\
=\frac{q b}{\alpha+\Phi^{(1)}\left(q+\xi_{1}\right)+\cdots+\Phi^{(m)}\left(q+\xi_{m}\right)}
\end{aligned}
$$

and, for $j=1, \ldots, m$, that

$$
\begin{aligned}
\mathrm{E}\left(\exp \left\{-\alpha T_{\tau}-\xi_{1} X^{(1)}\left(T_{\tau}-\right)-\cdots-\xi_{m} X^{(m)}\left(T_{\tau}-\right)-v \Delta_{\tau}\right\} \mathbf{1}_{A^{(j)}}\right) \\
=\left(\int_{0}^{\infty} \int_{[0, \infty)^{m}} \mathrm{e}^{-q\left(x_{1}+\cdots+x_{m}\right)} \mathrm{e}^{-\alpha a-\xi_{1} x_{1}-\cdots-\xi_{m} x_{m}} \mathrm{P}\left(X_{a}^{(1)} \in \mathrm{d} x_{1}\right) \cdots \mathrm{P}\left(X_{a}^{(m)} \in \mathrm{d} x_{m}\right) \mathrm{d} a\right) \\
\quad \times\left(\int_{(0, \infty)} \mathrm{e}^{-v z}\left(1-\mathrm{e}^{-q z}\right) \Pi^{(j)}(\mathrm{d} z)\right) \\
=\frac{\Phi^{(j)}(q+v)-\Phi^{(j)}(v)}{\alpha+\Phi^{(1)}\left(q+\xi_{1}\right)+\cdots+\Phi^{(m)}\left(q+\xi_{m}\right)} .
\end{aligned}
$$

In both cases, $\tau$ can be included in the joint transform in the same way as in the proof of Theorem 1(c).

Example 2. (Example 1 continued.) In the situation of Example 1, we have, e.g.

$$
\begin{gathered}
\mathrm{P}\left(T_{h} \in \mathrm{d} a, Y\left(T_{h}-\right) \in \mathrm{d} y, Z\left(T_{h}-\right) \in \mathrm{d} z, \Delta_{h} \in \mathrm{d} \zeta, A_{0}\right) \\
=\mathrm{P}\left(Y_{a} \in \mathrm{d} y, Z_{a} \in \mathrm{d} z \mid Y_{a}+Z_{a}=h\right) \mathrm{P}\left(H_{h} \in \mathrm{d} a\right) \delta_{0}(\mathrm{~d} \zeta), \\
\mathrm{P}\left(T_{h} \in \mathrm{d} a, Y\left(T_{h}-\right) \in \mathrm{d} y, Z\left(T_{h}-\right) \in \mathrm{d} z, \Delta_{h} \in \mathrm{d} \zeta, A_{Y}\right) \\
=\mathbf{1}_{\{y+z \leq h<y+z+\zeta\}} \mathrm{P}\left(Y_{a} \in \mathrm{d} y\right) \mathrm{P}\left(Z_{a} \in \mathrm{d} z\right) \mathrm{d} a \Pi_{Y}(\mathrm{~d} \zeta), \\
\mathrm{P}\left(T_{h} \in \mathrm{d} a, Y\left(T_{h}-\right) \in \mathrm{d} y, Z\left(T_{h}-\right) \in \mathrm{d} z, \Delta_{h} \in \mathrm{d} \zeta, A_{Z}\right) \\
=\mathbf{1}_{\{y+z \leq h<y+z+\zeta\}} \mathrm{P}\left(Y_{a} \in \mathrm{d} y\right) \mathrm{P}\left(Z_{a} \in \mathrm{d} z\right) \mathrm{d} a \Pi_{Z}(\mathrm{~d} \zeta) .
\end{gathered}
$$

Again, we can easily derive, from Theorem 2(c), the joint Laplace transform of all involved quantities, as in Corollary 1 . We leave the details to the reader, but mention that $\left(T_{\tau}, X^{(1)}\left(T_{\tau}-\right), \ldots, X^{(m)}\left(T_{\tau}-\right)\right)$ is independent of $\left(u_{\tau}, o_{\tau}\right)$, and that this has the following extension.

Proposition 3. $\left(X_{a}^{(0)}, \ldots, X_{a}^{(m)}\right)_{a<T_{\tau}}$ and $\left(u_{\tau}, o_{\tau}, \mathbf{1}_{A^{(0)}}, \ldots, \mathbf{1}_{A^{(m)}}\right)$ are independent.

Proof. The proof of Proposition 1 is easily adapted to the case of multiple subordinators.

Furthermore, we can characterize the law of $\left(T_{\tau}, X^{(1)}\left(T_{\tau}-\right), \ldots, X^{(m)}\left(T_{\tau}-\right)\right)$ as follows.

Proposition 4. $\left(T_{\tau}, X^{(1)}\left(T_{\tau}-\right), \ldots, X^{(m)}\left(T_{\tau}-\right)\right)$ is infinitely divisible with zero drift coeffcient and Lévy measure $a^{-1} \mathrm{e}^{-q x_{1}} \mathrm{P}\left(X_{a}^{(1)} \in \mathrm{d} x_{1}\right) \cdots \mathrm{e}^{-q x_{m}} \mathrm{P}\left(X_{a}^{(m)} \in \mathrm{d} x_{m}\right) \mathrm{d} a$. 
Proof. By Theorem 2(b), the law in question is given by

$$
\begin{aligned}
\mathrm{P}\left(T_{\tau} \in\right. & \left.\mathrm{d} a, X^{(1)}\left(T_{\tau}-\right) \in \mathrm{d} x_{1}, \ldots, X^{(m)}\left(T_{\tau}-\right) \in \mathrm{d} x_{m}\right) \\
& =\Phi(q) \mathrm{e}^{-q x_{1}} \mathrm{P}\left(X_{a}^{(1)} \in \mathrm{d} x_{1}\right) \cdots \mathrm{e}^{-q x_{m}} \mathrm{P}\left(X_{a}^{(m)} \in \mathrm{d} x_{m}\right) \mathrm{d} a \\
& =\Phi(q) \mathrm{e}^{-a \Phi(q)} \mathrm{P}\left(X_{a}^{(1, q)} \in \mathrm{d} x_{1}\right) \cdots \mathrm{P}\left(X_{a}^{(m, q)} \in \mathrm{d} x_{m}\right) \mathrm{d} a \\
& =\mathrm{P}\left(\tau(\Phi(q)) \in \mathrm{d} a, X^{(1, q)}(\tau(\Phi(q))) \in \mathrm{d} x_{1}, \ldots, X^{(m, q)}(\tau(\Phi(q))) \in \mathrm{d} x_{m}\right),
\end{aligned}
$$

where $X^{(j, q)}$ is constructed from $X^{(j)}$ by an exponential density transformation - see Section 33 of Sato [9] - which is also called temperation, discountation, or exponential tilting. The importance of this reformulation is that now the exponential random variable $\tau(\Phi(q))$ is independent of $\left(X^{(1, q)}, \ldots, X^{(m, q)}\right)$, and we may apply Lemma VI.7 of Bertoin [1] to obtain the Lévy measure

$$
\begin{aligned}
& a^{-1} \mathrm{e}^{-a \Phi(q)} \mathrm{P}\left(X_{a}^{(1, q)} \in \mathrm{d} x_{1}, \ldots, X_{a}^{(m, q)} \in \mathrm{d} x_{m}\right) \mathrm{d} a \\
& \quad=a^{-1} \mathrm{e}^{-q x_{1}} \mathrm{P}\left(X_{a}^{(1)} \in \mathrm{d} x_{1}\right) \cdots \mathrm{e}^{-q x_{m}} \mathrm{P}\left(X_{a}^{(m)} \in \mathrm{d} x_{m}\right) \mathrm{d} a,
\end{aligned}
$$

which completes the proof.

Finally, let us give two examples in which the joint densities can be calculated explicitly. Again, we consider the two-dimensional case, for simplicity. The extensions to higher dimensions are obvious.

Example 3. (Gamma subordinators.) Let $X_{a} \sim \Gamma\left(\vartheta_{X} a, m_{X}\right)$ and $Y_{a} \sim \Gamma\left(\vartheta_{Y} a, m_{Y}\right)$, i.e.

$$
\begin{aligned}
\frac{\mathrm{P}\left(X_{a} \in \mathrm{d} x\right)}{\mathrm{d} x} & =\frac{\left(x m_{X}\right)^{\vartheta_{X} a}}{\Gamma\left(\vartheta_{X} a\right)} \frac{\mathrm{e}^{-m_{X} x}}{x}, \\
\frac{\Pi_{X}(\mathrm{~d} z)}{\mathrm{d} z} & =\vartheta_{X} \frac{\mathrm{e}^{-m_{X} z}}{z}, \\
\Phi_{X}(q) & =\vartheta_{X} \ln \left(1+\frac{q}{m_{X}}\right),
\end{aligned}
$$

and similarly for $Y$. Then, by Theorem 2(a), $\left(T_{h}, X\left(T_{h}-\right), Y\left(T_{h}-\right), \Delta_{h}\right)$ has the joint density

$$
\begin{aligned}
\left.\mathbf{1}_{\{x+y} \leq h \leq x+y+z\right\} & \frac{m_{X}^{\vartheta_{X} a} m_{Y}^{\vartheta_{Y} a}}{\Gamma\left(\vartheta_{X} a\right) \Gamma\left(\vartheta_{Y} a\right)} \\
& \times x^{-\vartheta_{X} a-1} y^{-\vartheta_{Y} a-1} z^{-1} \mathrm{e}^{-m_{X} x-m_{Y} y}\left(\vartheta_{X} \mathrm{e}^{-m_{X} z}+\vartheta_{Y} \mathrm{e}^{-m_{Y} z}\right)
\end{aligned}
$$

on $[0, \infty)^{4}$, with some further simplifications if either or both of $\vartheta_{X}=\vartheta_{Y}$ and $m_{X}=m_{Y}$ hold. In particular, if $m_{X}=m_{Y}$ then $\left(\mathbf{1}_{A_{X}}, \mathbf{1}_{A_{Y}}\right)$ is independent of $\left(T_{\tau}, X\left(T_{\tau}-\right), Y\left(T_{\tau}-\right), \Delta_{\tau}\right)$ and

$$
\mathrm{P}\left(A_{X}\right)=\frac{\vartheta_{X}}{\vartheta_{X}+\vartheta_{Y}}=r, \quad \mathrm{P}\left(A_{Y}\right)=\frac{\vartheta_{Y}}{\vartheta_{X}+\vartheta_{Y}}=1-r .
$$

In fact, this is the case whenever $b_{X}=b_{Y}=0$ and $(1-r) \Pi_{X}=r \Pi_{Y}-$ not only in the case of gamma distributions. 
Example 4. (Inverse Gaussian subordinators.) Let $X_{a} \sim \operatorname{IG}\left(\delta_{X} a, \gamma_{X}\right)$ and $Y_{a} \sim \operatorname{IG}\left(\delta_{Y} a, \gamma_{Y}\right)$. This means that

$$
\begin{aligned}
\frac{\mathrm{P}\left(X_{a} \in \mathrm{d} x\right)}{\mathrm{d} x} & =\frac{\delta_{X} a}{\sqrt{2 \pi}} \mathrm{e}^{\gamma_{X} \delta_{X} a} \frac{\exp \left\{-\frac{1}{2} \gamma_{X}^{2} x-\frac{1}{2} \delta_{X}^{2} a^{2} x^{-1}\right\}}{x^{3 / 2}}, \\
\frac{\Pi_{X}(\mathrm{~d} z)}{\mathrm{d} z} & =\delta_{X} \frac{\gamma_{X}}{\sqrt{2 \pi}} \frac{\mathrm{e}^{-\gamma_{X}^{2} z}}{z^{3 / 2}}, \\
\Phi_{X}(q) & =\delta_{X}\left(\sqrt{\gamma_{X}^{2}+2 q}-\gamma_{X}\right),
\end{aligned}
$$

and, for $\gamma_{X}=\gamma_{Y}=\gamma$, this yields the following for the density of $\left(T_{h}, X\left(T_{h}-\right), Y\left(T_{h}-\right), \Delta_{h}\right)$ on $[0, \infty)^{4}$ :

$$
\begin{aligned}
\left.\mathbf{1}_{\{x+y} \leq h \leq x+y+z\right\} & \frac{\delta_{X} \delta_{Y} \gamma a^{2}}{(2 \pi)^{3 / 2}} \mathrm{e}^{\gamma\left(\delta_{X}+\delta_{Y}\right) a} \\
& \times \frac{\exp \left\{-\frac{1}{2} \gamma^{2}(x+y+z)-\frac{1}{2} \delta_{X}^{2} a^{2} x^{-1}-\frac{1}{2} \delta_{Y}^{2} a^{2} y^{-1}\right\}}{x^{3 / 2} y^{3 / 2} z^{3 / 2}}\left(\delta_{X}+\delta_{Y}\right) .
\end{aligned}
$$

\section{Applications in econometrics}

This section follows on from the model description of Section 2 and applies the main theoretical results of Section 4 in that context. Recall that Figure 1 shows how offers are represented in practice in electronic foreign-exchange markets. We add offers of realized transactions to this picture (passing from Figure 2(a) to Figure 2(b)) and think of the trajectories as realizations of stochastic processes. More precisely, we have price processes $\sigma$ and $\beta$ indexed by quantity in these figures and (by exchanging coordinate axes) quantity processes $S$ and $B$ indexed by price. We assume these processes to be subordinators (because they give us the freedom to choose the laws of the quantities traded in our model). The price $P=\inf \left\{a \geq 0: S_{a}>B_{a}\right\}$ is the equilibrium price at which transactions take place, $Q=S_{P}$ is the amount of currency that sellers offer at the market price $P$, and $Q^{\prime}=B_{P}$ is the amount of currency that buyers demand at price $P$. In general, $Q \neq Q^{\prime}$, i.e. either the last buyer or the last seller can only partially realize their transaction. We denote by $\Delta$ the total quantity offered or demanded. Clearly, the joint law of $\left(P, Q, Q^{\prime}, \Delta\right)$ is the central quantity in this setting.

Corollary 2. Let $B$ and $S$ be the subordinators associated to buyers' and sellers' offers via the expressions in (1).

(i) Assume that $B_{0}=h$ is a constant. Then,

$$
\begin{aligned}
\mathrm{P}(P \in & \left.\mathrm{d} a, Q \in \mathrm{d} x, Q^{\prime} \in \mathrm{d} y, \Delta \in \mathrm{d} z\right) \\
= & \mathbf{1}_{\{x<y, z \geq x-y\}} \mathrm{P}\left(S_{a} \in \mathrm{d} x\right) \mathrm{P}\left(B_{0}-B_{a}-z \in \mathrm{d} y\right) \mathrm{d} a \Pi_{\mathrm{B}}(\mathrm{d} z) \\
& +\mathbf{1}_{\{y<x, z \geq y-x\}} \mathrm{P}\left(S_{a}+z \in \mathrm{d} x\right) \mathrm{P}\left(B_{0}-B_{a} \in \mathrm{d} y\right) \mathrm{d} a \Pi_{\mathrm{S}}(\mathrm{d} z),
\end{aligned}
$$

where $\Pi_{\mathrm{B}}$ and $\Pi_{\mathrm{S}}$ are the respective Lévy measures of $B$ and $S$.

(ii) Assume that $B_{0}=\tau \sim \operatorname{Exp}(q)$ is independent of $B_{0}-B$ and $S$. Then

$$
\begin{aligned}
& \mathrm{E}\left(\exp \left\{-\alpha P-\xi Q-\eta Q^{\prime}-v \Delta\right\}\right) \\
& \quad=\frac{q\left(\Phi_{\mathrm{B}}(q+v)-\Phi_{\mathrm{B}}(v-\eta)+\Phi_{\mathrm{S}}(q+v+\eta+\xi)-\Phi_{\mathrm{S}}(v+\xi)\right)}{(q+\eta)\left(\alpha+\Phi_{\mathrm{B}}(q)+\Phi_{\mathrm{S}}(q+\eta+\xi)\right)},
\end{aligned}
$$

where $\Phi_{\mathrm{B}}$ and $\Phi_{\mathrm{S}}$ are the respective Laplace exponents of $B$ and $S$. 
Proof. The situation described is now the one in Examples 1 and 2, if we let $Y_{a}=S_{a}$ and $Z_{a}=h-B_{a}$. Note, however, that we have no drift components here: $b_{Y}=b_{Z}=b_{Y+Z}=0$. This leads to some simplifications; for example, the passage cannot take place continuously, i.e. $\mathrm{P}\left(A_{0}\right)=0$. In the notation of this section and the Introduction, we obtain the explicit laws of $\left(P, Q, Q^{\prime}, \Delta\right)$ as a corollary to Theorem 2 .

We can also split the formulae, as in Theorem 2, in order to specify whether $\Delta$ refers to a buyer or a seller. Also, by linear transformations, we can obtain other related quantities.

There are immediate extensions of the model away from homogeneity. One might consider higher intensities in a 'realistic' price range with decreasing tails or with cut-offs close to zero and infinity. This corresponds to a deterministic 'time change' of the subordinators. If both subordinators are transformed by the same time change, $T_{h}$ and associated quantities are just transformed accordingly. Some care is needed if the time change is not infinite - the agreed price may then be outside the model's allowed price range - which happens if the tails at infinity are integrable, particularly when cut off. Also, independent random time changes are a possibility. Dependent random time changes, different time changes for sellers and buyers, or replacing the subordinators by suitable and more general increasing additive processes lead out of the immediate range of the theory presented in this paper.

\section{Acknowledgements}

Modelling electronic foreign-exchange markets by subordinators was suggested to me by Neil Shephard. I am grateful to him and to Ole E. Barndorff-Nielsen for a number of stimulating discussions. Most of this research was carried out while the author was a visiting $\mathrm{PhD}$ student at MaPhySto (Centre for Mathematical Physics and Stochastics, funded by The Danish National Research Foundation) at the University of Aarhus.

I thank Clive Bowsher for pointing out to me empirical work in the econometrics literature, and an anonymous referee for suggesting some improvements to the paper's presentation.

This research was supported by a PhD scholarship from the DAAD (German Academic Exchange Service) within the scope of the common programme HSP III of the German Federal and Länder Governments.

\section{References}

[1] Bertoin, J. (1996). Lévy Processes. Cambridge University Press.

[2] Bertoin, J. (1999). Subordinators: Examples and Applications (Ecole d'été de Probabilités de St-Flour XXVII; Lecture Notes Math. 1717). Springer, Berlin.

[3] Bowsher, C. (2004). Dynamic latent spline models for the bid and ask curves of an electronic limit order book market. Work in progress, University of Oxford.

[4] Goodhart, C. (1998). The foreign exchange market - a visual representation. LSE Video 0198, VHS 38 mins.

[5] Gouriéroux, C., Le Fol, G. and Meyer, B. (1998). Etude du carnet d'ordres. Banque et Marchés 36, 5-20.

[6] GusaK, D. V. (1969). On the joint distribution of the first exit time and exit value for homogeneous processes with independent increments. Theory Prob. Appl. 14, 14-23.

[7] Kesten, H. (1969). Hitting Probabilities of Single Points for Processes with Stationary Independent Increments (Memoirs Am. Math. Soc. 93). American Mathematical Society, Providence, RI.

[8] Revuz, D. AND Yor, M. (1999). Continuous Martingales and Brownian Motion, 3rd edn. Springer, Berlin.

[9] Sato, K. (1999). Lévy Processes and Infinitely Divisible Distributions. Cambridge University Press.

[10] Winkel, M. (2001). Electronic foreign exchange markets and level passage events of multivariate subordinators. Res. Rep. 41, MaPhySto. Available at http://www.maphysto.dk/oldpages/publications/.

[11] Winkel, M. (2001). Some contributions to the theory of Lévy processes and applications in turbulence and econometrics. Doctoral Thesis, Université Pierre et Marie Curie. 